\title{
A RARE CASE REPORT OF PARATESTICULAR RHABDOMYOSARCOMA IN AN ADULT PATIENT
}

\author{
Chandumal, Mussadique Ali, Manzoor Khan, Jhanzeb Iftikhar, Samia Yasmeen, Umm e Kalsoom Awan \\ Shaukat Khanum Memorial Cancer Hospital and Research Centre, Lahore Pakistan
}

\begin{abstract}
Rhabdomyosarcoma is a malignancy of mesenchymal tissue origin typically occurring in childhood and adolescence, with an incidence rate of of 4.3 patients per million population per anum. Primary rhabdomyosarcoma of para-testicular origin is an infrequent condition, making up to $7 \%$ of all the cases of rhabdomyosarcoma tumors in children and adolescents. Here we describe a case of a 25 years old man with left paratesticular solid mass. He underwent left sided orchiectomy with histopathology revealing paratesticuler embryonal rhabdomyosarcoma. He underwent definitive chemo-radiotherapy and orchidopexy with complete remission and is on active surveillance for 4 years.
\end{abstract}

Keywords: Paratesticular, Remission, Rhabdomyosarcoma.

This is an Open Access article distributed under the terms of the Creative Commons Attribution License (http://creativecommons.org/licenses/by/4.0), which permits unrestricted use, distribution, and reproduction in any medium, provided the original work is properly cited.

\section{INTRODUCTION}

Rhabdomyosarcoma (RMS) is the commonest soft tissue sarcoma of childhood and adolescence, with an incidence rate of around $5 \%$ cases per million children per year, aged under 15 years $^{1}$. RMS is of 3 major histologic subtypes: Embryonal (59\%), Alveolar (20-30\%) and Undifferentiated $(20 \%)^{2}$.

Para-testicular RMS is an extremely rare condition, with only few reported cases in literature 3 . It has a high malignant potential with rapid spread, and requiring early diagnosis and treatment, especially in the aged patients, where prognosis is worse ${ }^{4}$. Embryonal RMS presents as a large painless, palpable mass ( $>5$ $\mathrm{cm})$, with no distinction between benign and malignant tumors at times ${ }^{5}$. The preferred surgical approach is a wide incision via inguinal approach and a high ligation of the testicle and the spermatic cord 6 . A case series of six patients of primary para-testicular RMS in adult patients has been published recently where surgery and chemotherapy resulted in a reduced local recurrence, an improvementin disease-free and overall survival rate in adult patients with distant metastases ${ }^{3}$.

This aim of presenting this case report is to highlight clinical presentation and management of paratesticular RMS in in adult patient.

\section{CASE REPORT}

Our patient is a 25 years old man with history of left testicular painless swelling for 3 months. Ultrasound scrotum revealed hypoechoic left scrotal mass

Correspondence: Dr Mussadique Ali, Medical Oncology Department, SKMCH \& RC, Lahore Pakistan

Received: 06 Jul 2020; revised received: 11 Aug 2020; accepted: 17 Aug 2020 with calcifications, and ultrasound scan of abdomen and pelvis revealed normal intraabdominal organs sonologically. He underwent left inguinal orchiectomy at some other hospital and histopathology revealed Para testicular embryonal rhabdomyosarcoma. He was then referred to our hospital, where staging work up revealed multiple enlarged lobulated retroperitoneal lymph nodes seen largest around left para arotic recess measuring $4.2 \mathrm{~cm}$ (figure). Alphafetoprotein (AFP), beta human chorionic gonadotropin (Beta-hCG) and serum lactate dehydrogenase (LDH) were normal. As per risk stratification for non-metastatic RMS study, he was grouped in high risk subgroup. His case was discussed in weekly sarcoma MDT and was offered treatment with chemotherapy. This was succ-eeded by retroperitoneal lymphadenectomy and che-moradiotherapy.

He received four cycles of of IVA-DO (vincristine, Dactinomycin, and doxorubicin and Ifosphomide) followed by left retroperitoneal (left paraarotic, aortocaval) lymph node dissection. The histopathology revealed involvement of 2 out of 24 lymph nodes positive for rhabdomyosarcoma, with largest deposit measuring $1.5 \mathrm{~cm}$ and testicular vein resection margins were free of tumor. Orchidopexy with transposition of right testis to right anterior abdominal wall was then contemplated to reduce the risk of permanent aspermia. He then underwent chemoradiotherapy with dose of $41.4 \mathrm{G}$ in 23 fractions along with five cycles of IVA (Ifosphomide, vincristine and dactinomycin). End of treatment scan revealed no evidence of local disease, lymphadenopathy or distant metastases. Currently he is on active surveillance with no evidence of disease recurrence four years after treatment. 


\section{DISCUSSION}

Briefly, our case is primary para-testicular RMS, treated with orchiectomy and retroperitoneal lymphadenectomy followed by definitive chemo-radiotherapy with sustained remission for 4 years.

Para-testicular RMS is an infrequent malignant
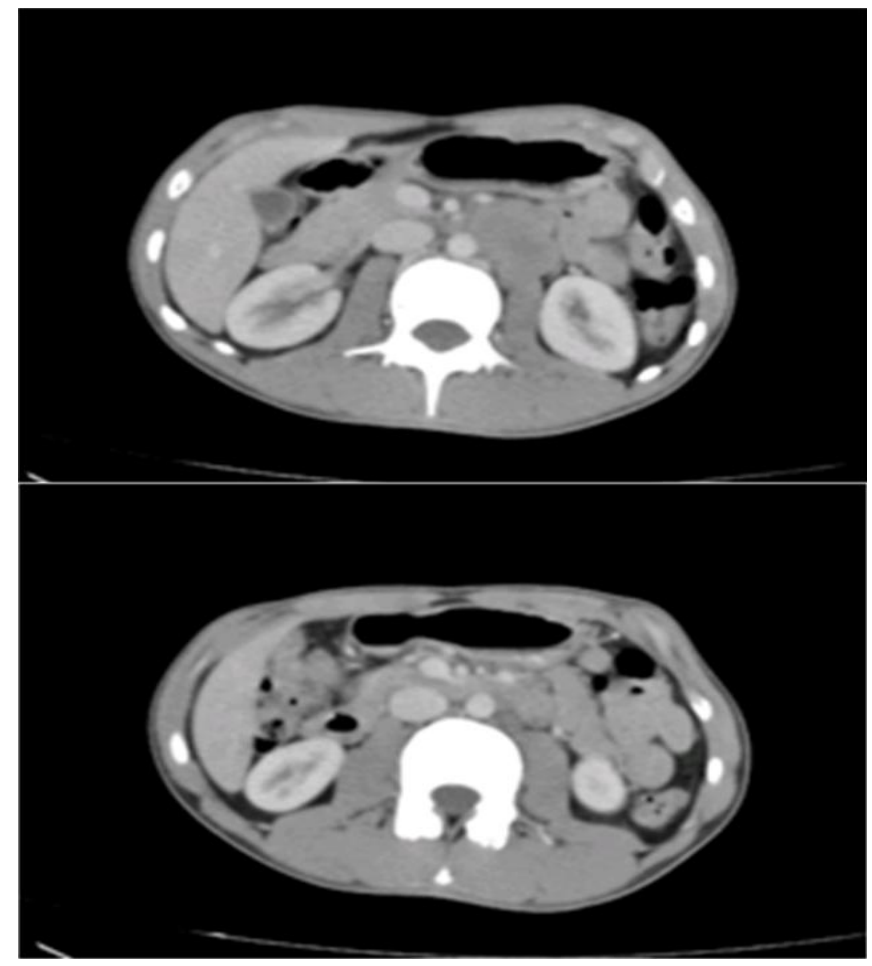

Figure-1: Baseline axial CT images showing left paraaortic nodes.

condition where tumor develops within the mesenchymal tissues of the epididymis and the spermatic cord. Tumor markers including AFP, beta-hCG, and LDH are usually normal. At initial clinical presentation, para-testicular RMS often presents with nodal involvement or metastases. Embryonal subtype of RMS is the most frequent histology and the typical site for its occurrence is the head, neck, the genitalia and the urinary tract, yet, it may arise at any primary site 7 . Main mode of distant dissemination of this tumor is by the lymphatic pathway to the iliac nodes and the paraaortic nodes, but blood born dissemination also occurs with most common distant site being lungs and liver 8 . Radical surgery is mandatory for all patients with complete surgical resection of the primary tumor and when there is disease in retroperitoneal lymph nodes, retroperitoneal lymphadenectomy is recommended 9 .

Chemotherapy is routinely prescribed since rhabdomyosarcoma is a chemosensitive disease and radio- therapy has a role complementary to chemotherapy in eliminating remaining disease, local as well as retroperitoneal lymph nodes ${ }^{10}$. The combination of all three modalities, that is complete surgical resection, pharmacotherapy and radiotherapy have greatly enhanced the survival rate in para-testicular RMS with no significant long-term complications.

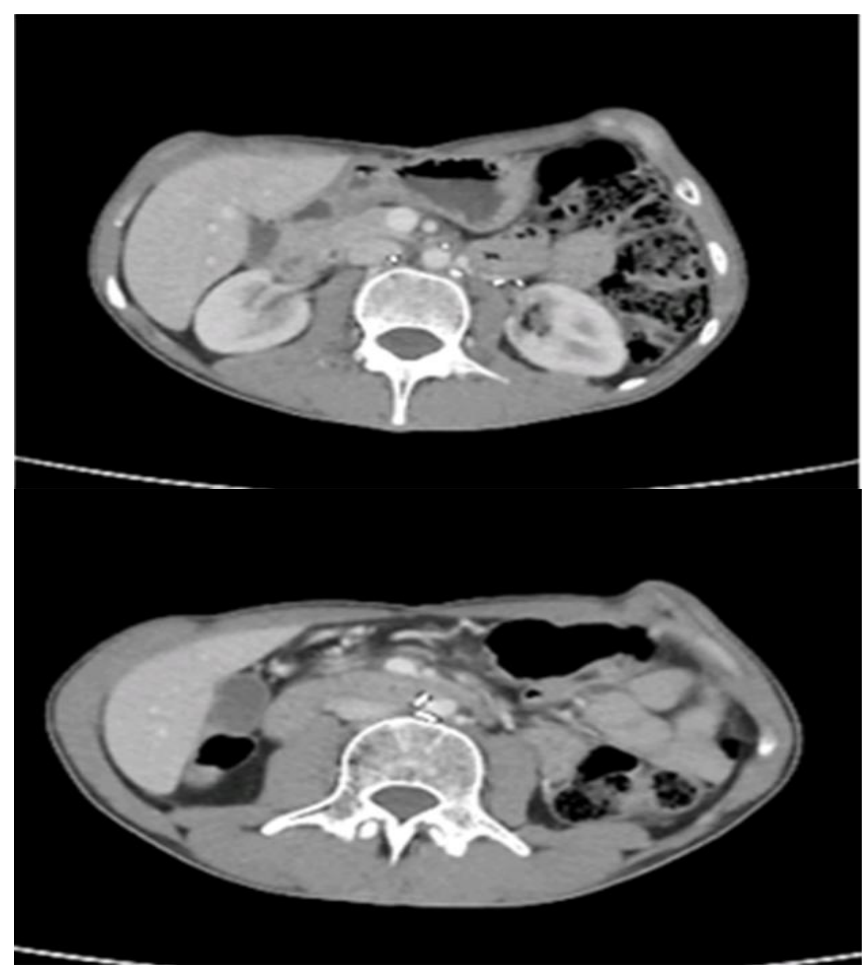

Figure-2: End of treatment scan showing complete resolution of nodal disease.

\section{CONCLUSION}

Para-testicular RMS is a rare and particularly aggressive disease, manifesting primarily in children and adolescents but can rarely affect adults as well. When localized, this disease has a good prognosis and where metastatic, it has a very dismal outcome. A well planned and timely treatment based on surgery and chemotherapy yields good results. Radiotherapy, when indicated in case of residual local disease foci and retroperitoneal lymph nodes also plays very important role in management. RMS patients need strict follow up to detect early recurrences.

\section{CONFLICT OF INTEREST}

This study has no conflict of interest to be declared by any author.

\section{REFERENCES}

1. Dagher R, Helman L. Rhabdomyosarcoma: an overview. Oncologist 1999; 4(1): 34-44. 
2. Goldfarb B, Khoury AE, Greenberg ML, Churchill BM, Smith CR, McLorie GA. The role of retroperitoneal lymphadenectomy in localized paratesticular rhabdomyosarcoma. J Urol 1994; 152 (2 Pt 2): 785-87.

3. Stewart LH, Lioe TF, Johnston SR. Thirty-year review of intrascrotal rhabdomyosarcoma. Br J Urol 1991; 68(4): 418-20.

4. Haga K, Kashiwagi A, Nagamori S, Yamashiro K. Adult paratesticular rhabdomyosarcoma. Nat Clin Pract Urol 2005; 2(8): 398403.

5. Mejía-Salas JA, Sánchez-Corona H, Priego-Niño A, CárdenasRodríguez E, Sánchez-Galindo JA. Rabdomiosarcoma testicular primario: reporte de un caso. Primary testicular rhabdomyosarcoma: A case report. Cir Cir 2017; 85(2): 143-47.

6. Hammond WJ, Farber BA, Price AP, Wolden SL, Heaton TE,
Wexler LH, et al. Paratesticular rhabdomyosarcoma: Importance of initial therapy. J Pediatr Surg 2017; 52(2): 304-08.

7. Bisogno G, Jenney M, Bergeron C, Melcon SG, Ferrari A, Oberlin $\mathrm{O}$, et al. Addition of dose-intensified doxorubicin to standard chemotherapy for rhabdomyosarcoma (EpSSG RMS 2005): a multicentre, open-label, randomised controlled, phase 3 trial. Lancet Oncol. 2018; 19(8): 1061-71.

8. Meyer WH, Spunt SL. Soft tissue sarcomas of childhood. Cancer Treat Rev 2004; 30(3): 269-80.

9. Leuschner I. Spindle cell rhabdomyosarcoma: histologic variant of embryonal rhabdomyosarcoma with association to favorable prognosis. Curr Top Pathol 1995; 89: 261-72.

10. Maurer HM, Beltangady M, Gehan EA. The Intergroup Rhabdomyosarcoma Study-I. A final report. Cancer 1988; 61(2): 209-20. 